\title{
Jump loci in the equivariant spectral sequence
}

\author{
Stefan Papadima And Alexander I. Suciu
}

\begin{abstract}
We study the homology jump loci of a chain complex over an affine $\mathbb{k}$-algebra. When the chain complex is the first page of the equivariant spectral sequence associated to a regular abelian cover of a finite-type CW-complex, we relate those jump loci to the resonance varieties associated to the cohomology ring of the space. As an application, we show that vanishing resonance implies a certain finiteness property for the completed Alexander invariants of the space. We also show that vanishing resonance is a Zariski open condition, on a natural parameter space for connected, finitedimensional commutative graded algebras.
\end{abstract}

\section{Introduction}

\subsection{Overview}

The study of the homology groups of abelian covers goes back to the $1920 \mathrm{~s}$, when J.W. Alexander introduced his eponymous knot polynomial. Given a knot in $S^{3}$, let $X^{\mathrm{ab}} \rightarrow X$ be the universal abelian cover of its complement. The Alexander polynomial of the knot, then, is the order of $H_{1}\left(X^{\mathrm{ab}}, \mathbb{Z}\right)$, a finitely generated, torsion module over the group-ring $\mathbb{Z}\left[H_{1}(X, \mathbb{Z})\right] \cong \mathbb{Z}\left[t^{ \pm 1}\right]$.

More generally, consider a connected, finite-type CW-complex $X$, with fundamental group $\pi=\pi_{1}\left(X, x_{0}\right)$. The homology groups of $X^{\mathrm{ab}}$, with coefficients in $\mathbb{C}$, are finitely generated modules over the Noetherian ring $\mathbb{C}\left[\pi_{\mathrm{ab}}\right]$. As shown by Dwyer and Fried in [5], the support loci of the Alexander invariants $H_{i}\left(X^{\mathrm{ab}}, \mathbb{C}\right)$ completely determine the (rational) homological finiteness properties of its regular, free-abelian covers. This result was recast in [11] in terms of the characteristic varieties of $X$, defined as the jump loci for homology with coefficients in rank 1 local systems on our space. For a

2000 Mathematics Subject Classification. Primary 55N25. Secondary 14M12, 20J05, 55T99.

Key words and phrases. affine algebra, maximal spectrum, homology jump loci, support varieties, equivariant spectral sequence, resonance variety, characteristic variety, Alexander invariants, completion. 
detailed treatment of these topics, and further developments, we refer to $[14,15]$.

We revisit here this theory from a more general point of view. The key new ingredient in our approach is the equivariant spectral sequence from [10]. Using techniques from commutative algebra, we establish a tight connection between the homology jump loci of the first page of this spectral sequence, on one hand, and the resonance varieties associated to the cohomology ring of the space, on the other hand. In turn, this connection allows us to prove an infinitesimal analog of the Dwyer-Fried finiteness test for the completed homology groups of abelian Galois covers.

\subsection{The equivariant spectral sequence}

Let $\nu: \pi \rightarrow G$ be an epimorphism from the fundamental group of $X$ to a (finitely generated) abelian group $G$, and let $X^{\nu} \rightarrow X$ be the corresponding regular cover. The homology groups of $X^{\nu}$ with coefficients in an algebraically closed field $\mathbb{k}$ are modules over the group-ring $\mathbb{k} G$.

Let $J$ be the augmentation ideal of $\mathbb{k} G$. The associated graded ring, $S=\operatorname{gr}_{J}(\mathbb{k} G)$, then, is a finitely generated $\mathbb{k}$-algebra. As shown in [10], there is a spectral sequence over $S$ that converges to the $J$-adic completion of $H_{*}\left(X^{\nu}, \mathbb{k}\right)$. The first page of this spectral sequence is a chain complex $E$, with terms the finitely generated $S$-modules $E_{i}=S \otimes_{\mathbb{k}} H_{i}(X, \mathbb{k})$ and differentials that can be written in terms of the co-multiplication in $H_{*}(X, \mathbb{k})$ and the induced homomorphism $\nu_{*}: H_{1}(\pi, \mathbb{k}) \rightarrow H_{1}(G, \mathbb{k})$.

The homology jump loci of $E$ are the subsets $\mathcal{V}_{d}^{i}(E)$ of the maximal spectrum $\operatorname{Spec}(S)$ consisting of those maximal ideals $\mathfrak{m}$ for which the $\mathbb{k}$ vector space $H_{i}\left(E \otimes_{S} S / \mathfrak{m}\right)$ has dimension at least $d$. To understand these sets in a more geometric fashion, consider the projection $\phi: G \rightarrow \bar{G}$ onto the maximal free-abelian quotient of $G$. Then, as we show in Lemma 5.1, the set $\operatorname{Spec}(S)$ may be identified with $H^{1}(\bar{G}, \mathbb{k})$. Thus, we may view the sets $\mathcal{V}_{d}^{i}(E)$ as subvarieties of the $\mathbb{k}$-vector space $H^{1}(\bar{G}, \mathbb{k})$.

The above definition of homology jump loci works for arbitrary chain complexes $E$ over a finitely generated $\mathbb{k}$-algebra $S$. In the case when $E$ is the cellular chain complex of the universal abelian cover $X^{\mathrm{ab}}$, with coefficients in $\mathbb{k}$, and $S=\mathbb{k}\left[\pi_{\mathrm{ab}}\right]$, the corresponding jump loci (also known as the characteristic varieties of $X$ ) are Zariski closed subsets of the character group $\operatorname{Hom}\left(\pi_{\mathrm{ab}}, \mathbb{k}^{\times}\right)$. These sets, which were introduced by Green and Lazarsfeld in [8], are extremely useful in a variety of settings, see for instance $[2,4,11,14,15]$. 


\subsection{Resonance varieties}

To state our main result, we need to recall one more concept. Using the cohomology algebra $A=H^{*}(X, \mathbb{k})$ as input, we define the resonance varieties of $X$ as the sets $\mathcal{R}_{d}^{i}(X, \mathbb{k})$ consisting of those square-zero elements $a \in A^{1}$ for which the $\mathbb{k}$-vector space $H^{i}(A, a)$ has dimension at least $d$, where $(A, a)$ is the cochain complex with terms $A^{i}$ and differentials given by leftmultiplication by $a$. As we show in Corollary 4.2 , it is enough to assume that $X$ has finite $k$-skeleton, in order to conclude that the sets $\mathcal{R}_{d}^{i}(X, \mathbb{k})$ are Zariski closed, for all $i \leq k$ and $d>0$.

The vanishing property for resonance plays a crucial role in Theorem 1.1(2) and Corollary 1.2 below. In Sections 3.2 and 3.3, which are largely selfcontained and independent of the rest of the paper, we analyze this vanishing property for arbitrary commutative graded algebras. More precisely, we show in Theorem 3.3 that, if $B$ is a connected, finite-dimensional, graded $\mathbb{k}$-vector space and $P_{B}$ is the parameter space for all commutative graded algebras whose underlying graded vector space is $B$, then the subset of those cgas whose resonance vanishes is a Zariski open subset of $P_{B}$.

\subsection{Resonance and the completed Alexander invariants}

We are now ready to state our main result, which relates the resonance varieties of a space to the finiteness properties of the completed homology groups of its abelian Galois covers. (Proofs for the two parts of this result will be given in Theorems 5.2 and 6.2 , respectively.)

Theorem 1.1. Let $X$ be a connected $C W$-complex, let $\nu: \pi \rightarrow G$ be a homomorphism onto a finitely generated abelian group $G$, and let $\bar{\nu}^{*}: H^{1}(\bar{G}, \mathbb{k}) \hookrightarrow$ $H^{1}(\pi, \mathbb{k})$ be the homomorphism induced in cohomology by $\bar{\nu}=\phi \circ \nu$.

(1) If $X$ is of finite type, and $E$ is the first page of the corresponding equivariant spectral sequence, then $\bar{\nu}^{*}\left(\mathcal{V}_{d}^{i}(E)\right)=\operatorname{im}\left(\bar{\nu}^{*}\right) \cap \mathcal{R}_{d}^{i}(X, \mathbb{k})$, for all $i \geq 0$ and $d>0$.

(2) Suppose $X$ has finite $k$-skeleton for some $k \geq 1$, and, for each $0 \leq i \leq$ $k$, the linear subspace $\operatorname{im}\left(\bar{\nu}^{*}\right)$ intersects the resonance variety $\mathcal{R}_{1}^{i}(X, \mathbb{k})$ at most at 0 . Then, for each $0 \leq i \leq k$, the completion of $H_{i}\left(X^{\nu}, \mathbb{k}\right)$ with respect to the $J$-adic filtration is finite dimensional.

As an application, we obtain the following corollary. 
Corollary 1.2. If $X$ has finite $k$-skeleton, and all the resonance varieties $\mathcal{R}_{1}^{i}(X, \mathbb{k})$ with $i \leq k$ vanish or are empty, then the completions of the Alexander invariants $H_{i}\left(X^{\mathrm{ab}}, \mathbb{k}\right)$ are finite dimensional $\mathbb{k}$-vector spaces, for all $i \leq k$.

This corollary generalizes Theorem $\mathrm{C}(2)$ from [2], a result proved in that paper in the case when $k$ equals 1 and the coefficient field is $\mathbb{C}$, using a different approach. It should be noted that, even in this situation, the Alexander invariants themselves may well be infinite dimensional.

An interesting example where Corollary 1.2 applies arises when $X=\mathcal{T}_{g}$, the Torelli space for curves of genus $g \geq 4$. Working over $\mathbb{C}$, the vanishing resonance property for $k=1$ is proved in [2, Theorem 4.4]. Furthermore, Theorem B from [1], in genus $g \geq 6$, together with a recent result of Hain from [9], in genus $g=4$ or 5 , give a non-trivial computation of the completion of $H_{1}\left(\mathcal{T}_{g}^{\mathrm{ab}}, \mathbb{C}\right)$.

\section{The homology jump loci of a chain complex}

In this section, we introduce the support varieties and homology jump loci of a chain complex over a finitely generated $\mathbb{k}$-algebra, and study some of their properties.

\subsection{Maximal spectrum and supports}

We start by reviewing some standard notions from commutative algebra, as they can be found, for instance, in [6].

Fix a ground field $\mathbb{k}$. Let $S$ be a commutative, finitely generated $\mathbb{k}$ algebra (also known as an affine $\mathbb{k}$-algebra), and let $\operatorname{Spec}(S)$ be the maximal spectrum of $S$. This set comes endowed with the Zariski topology, whereby a subset $F$ is closed if and only if there is an ideal $\mathfrak{a} \subseteq S$ such that $F$ equals $V(\mathfrak{a})=\{\mathfrak{m} \mid \mathfrak{m} \supseteq \mathfrak{a}\}$, the zero set of the ideal $\mathfrak{a}$.

Now suppose $\mathbb{k}$ is algebraically closed. Then $S / \mathfrak{m}=\mathbb{k}$, for every maximal ideal $\mathfrak{m}$, and we have a natural identification

$$
\operatorname{Spec}(S)=\operatorname{Hom}_{\mathbb{k} \text {-alg }}(S, \mathbb{k}),
$$

under which a maximal ideal $\mathfrak{m} \subset S$ corresponds to the $\mathbb{k}$-algebra morphism $\rho: S \rightarrow S / \mathfrak{m}=\mathbb{k}$. 
Denote by $S_{\mathfrak{m}}$ the localization of the ring $S$ at the maximal ideal $\mathfrak{m}$. Clearly, the above morphism $\rho$ factors through a ring morphism $\rho_{\mathfrak{m}}: S_{\mathfrak{m}} \rightarrow$ $S_{\mathfrak{m}} / \mathfrak{m} S_{\mathfrak{m}}$. Given an $S$-module $M$, denote by $M_{\mathfrak{m}}$ its localization at the maximal ideal $\mathfrak{m}$; then $M_{\mathfrak{m}}$ acquires in a natural way the structure of an $S_{\mathfrak{m}}$-module. Define the support of $M$ as

$$
\operatorname{supp}(M)=\left\{\mathfrak{m} \in \operatorname{Spec}(S) \mid M_{\mathfrak{m}} \neq 0\right\}
$$

If $M$ is a finitely generated $S$-module, the support of $M$ is a Zariski closed subset of $\operatorname{Spec}(S)$, since

$$
\operatorname{supp}(M)=V(\operatorname{ann}(M)),
$$

where $\operatorname{ann}(M) \subseteq S$ is the annihilator $M$.

\subsection{Support loci}

Let $E=\left(E_{\bullet}, d\right)$ be a non-negatively graded chain complex over $S$; in other words, a sequence of $S$-modules $\left\{E_{i}\right\}_{i \geq 0}$ and $S$-linear maps between them,

$$
\cdots \longrightarrow E_{i} \stackrel{d_{i}}{\longrightarrow} E_{i-1} \longrightarrow \cdots \longrightarrow E_{1} \stackrel{d_{1}}{\longrightarrow} E_{0} \longrightarrow 0
$$

satisfying $d_{i} \circ d_{i+1}=0$. Evidently, the homology groups of the chain complex, $H_{i}(E)=\operatorname{ker} d_{i} / \operatorname{im} d_{i+1}$, are again $S$-modules.

Definition 2.1. The support varieties of the $S$-chain complex $E$ are the supports of the exterior powers of the homology modules $H_{*}(E)$ :

$$
\mathcal{W}_{d}^{i}(E)=\operatorname{supp}\left(\bigwedge^{d} H_{i}(E)\right) .
$$

These subsets of $\operatorname{Spec}(S)$ are defined for all integers $i$ and all nonnegative integers $d$; they are empty if $i<0$ and $d>0$. Furthermore, for each $i \geq 0$, we have a nested sequence

$$
\operatorname{Spec}(S)=\mathcal{W}_{0}^{i}(E) \supseteq \mathcal{W}_{1}^{i}(E) \supseteq \mathcal{W}_{2}^{i}(E) \supseteq \cdots
$$

It is readily checked that these sets depend only on the chain-homotopy equivalence class of the $S$-chain complex $E$.

Now suppose $E$ is a chain complex of finitely generated $S$-modules. Then the sets $\mathcal{W}_{d}^{i}(E)$ are Zariski closed subsets of $\operatorname{Spec}(S)$, for all integers $i$ and 
$d \geq 0$. Indeed, if $E_{i}$ is a finitely generated $S$-module, then $H_{i}(E)$ is also finitely generated, and so are all its exterior powers; thus, the assertion follows from formula (3).

\subsection{Homology jump loci}

Let $E$ be a chain complex of $S$-modules as in (4). From now on, we will assume that the coefficient field $\mathbb{k}$ is algebraically closed, to insure that the residue fields $S / \mathfrak{m}$ are isomorphic to $\mathbb{k}$, for all $\mathfrak{m} \in \operatorname{Spec}(S)$.

Definition 2.2. The homology jump loci of the $S$-chain complex $E$ are defined as

$$
\mathcal{V}_{d}^{i}(E)=\left\{\mathfrak{m} \in \operatorname{Spec}(S) \mid \operatorname{dim}_{\mathbb{k}} H_{i}\left(E \otimes_{S} S / \mathfrak{m}\right) \geq d\right\} .
$$

As before, these sets are defined for all integers $i$ and all non-negative integers $d$; they are empty if $i<0$ and $d>0$; and, for each $i \geq 0$, they form a nested sequence

$$
\operatorname{Spec}(S)=\mathcal{V}_{0}^{i}(E) \supseteq \mathcal{V}_{1}^{i}(E) \supseteq \mathcal{V}_{2}^{i}(E) \supseteq \cdots .
$$

Furthermore, the sets $\mathcal{V}_{d}^{i}(E)$ depend only on the chain-homotopy equivalence class of $E$. Under some mild restrictions on the chain complex $E$, its homology jump loci are Zariski closed subsets. The next lemma makes this statement more precise.

Lemma 2.3. Suppose $E$ is a chain complex of free, finitely generated $S$ modules, and $\mathbb{k}$ is algebraically closed. Then the sets $\mathcal{V}_{d}^{i}(E)$ are Zariski closed subsets of $\operatorname{Spec}(S)$, for all integers $i$ and $d \geq 0$.

Proof. By definition, a maximal ideal $\mathfrak{m} \in \operatorname{Spec}(S)$ belongs to the set $\mathcal{V}_{d}^{i}(E)$ if and only if $\operatorname{rank} d_{i+1}(\mathfrak{m})+\operatorname{rank} d_{i}(\mathfrak{m}) \leq c_{i}-d$, where $c_{i}=\operatorname{rank}_{S} E_{i}$ and $d_{i}(\mathfrak{m})=d_{i} \otimes_{S} S / \mathfrak{m}$. Hence, $\mathcal{V}_{d}^{i}(E)$ is the zero-set of the ideal generated by all minors of size $c_{i}-d+1$ of the block-matrix $d_{i+1} \oplus d_{i}$.

As the next example indicates, the freeness assumption from Lemma 2.3 is crucial for the conclusion to hold, even in the presence of the finitegeneration assumption. We refer to [3, Example 9.5] for other, more exotic examples, where both the freeness and the finite-generation assumptions are violated. 
Example 2.4. Let $\mathbb{k}$ be an algebraically closed field, and let $S=\mathbb{k}[x]$. Consider the chain complex of (finitely-generated) $S$-modules $E: S \stackrel{\epsilon}{\longrightarrow} \mathbb{k}$, where $E_{0}=\mathbb{k}$, viewed as a trivial $S$-module, and $\epsilon$ is the $\mathbb{k}$-algebra map given by $\epsilon(x)=0$. It is readily verified that $\mathcal{V}_{1}^{1}(E)=\mathbb{k} \backslash\{0\}$, which, of course, is not a Zariski closed subset of $\operatorname{Spec}(S)=\mathbb{k}$.

\subsection{Comparing the two sets of loci}

We are particularly interested in the sets $\mathcal{W}_{1}^{i}(E)=\operatorname{supp} H_{i}(E)$ and $\mathcal{V}_{1}^{i}(E)=$ $\left\{\mathfrak{m} \mid H_{i}\left(E \otimes_{S} S / \mathfrak{m}\right) \neq 0\right\}$. The next theorem (which, as we shall see in Section 4.3, generalizes a result from [11]), establishes a comparison between these two types of sets.

Theorem 2.5. Let $S$ be a finitely generated algebra over an algebraically closed field $\mathbb{k}$, and let $E$ be a chain complex of free, finitely generated $S$-modules. Then, for all integers $q$,

$$
\bigcup_{i \leq q} \mathcal{W}_{1}^{i}(E)=\bigcup_{i \leq q} \mathcal{V}_{1}^{i}(E)
$$

Proof. If $q \leq-1$, we have $\bigcup_{i \leq q} \mathcal{W}_{1}^{i}(E)=\bigcup_{i \leq q} \mathcal{V}_{1}^{i}(E)=\emptyset$. The case $q \geq-1$ is done by induction on $q$, starting at $q=-1$.

Let $\mathfrak{m}$ be a maximal ideal in $S$, and let $\rho: S \rightarrow S / \mathfrak{m}$ be the corresponding $\mathbb{k}$-algebra morphism. Consider the Künneth spectral sequence associated to the free chain complex $E \otimes_{S} S_{\mathfrak{m}}$ and the change-of-rings map $\rho_{\mathfrak{m}}: S_{\mathfrak{m}} \rightarrow$ $S_{\mathfrak{m}} / \mathfrak{m} S_{\mathfrak{m}}$ :

$$
E_{s, t}^{2}=\operatorname{Tor}_{s}^{S_{\mathfrak{m}}}\left(H_{t}(E)_{\mathfrak{m}}, S_{\mathfrak{m}} / \mathfrak{m} S_{\mathfrak{m}}\right) \Rightarrow H_{s+t}\left(E \otimes_{S} S / \mathfrak{m}\right)
$$

First suppose $\mathfrak{m} \notin \bigcup_{i=0}^{q} \mathcal{W}_{1}^{i}(E)$. Then, for each $t \leq q$, we have $H_{t}(E)_{\mathfrak{m}}=$ 0 , and so $E_{s, t}^{2}=0$, and thus $E_{s, t}^{\infty}=0$. Consequently, $H_{i}\left(E \otimes_{S} S / \mathfrak{m}\right)=0$, for all $i \leq q$, which means that $\mathfrak{m} \notin \bigcup_{i=0}^{q} \mathcal{V}_{1}^{i}(E)$.

Now suppose $\mathfrak{m} \in \bigcup_{i=0}^{q} \mathcal{W}_{1}^{i}(E)$. We may assume $\mathfrak{m} \notin \operatorname{supp} H_{t}(E)$, for all $t<q$, and $\mathfrak{m} \in \operatorname{supp} H_{q}(E)$, for otherwise we will be done, by the induction hypothesis. These assumptions mean that $H_{t}(E)_{\mathfrak{m}}=0$, for $t<q$, and $H_{q}(E)_{\mathfrak{m}} \neq 0$. In particular, we have $E_{s, t}^{2}=0$, for $t<q$, and thus $E_{0, q}^{2}=E_{0, q}^{\infty}$. Hence,

$$
\begin{aligned}
H_{q}\left(E \otimes_{S} S / \mathfrak{m}\right) & =E_{0, q}^{\infty}=E_{0, q}^{2} \\
& =H_{q}(E)_{\mathfrak{m}} \otimes_{S_{\mathfrak{m}}} S_{\mathfrak{m}} / \mathfrak{m} S_{\mathfrak{m}}=H_{q}(E)_{\mathfrak{m}} / \mathfrak{m} H_{q}(E)_{\mathfrak{m}}
\end{aligned}
$$


By assumption, $E_{q}$ is a finitely generated $S$-module; thus, $H_{q}(E)$ is also finitely generated. Hence, $H_{q}(E)_{\mathfrak{m}}$ is a non-zero, finitely generated $S_{\mathfrak{m}}$-module. By Nakayama's Lemma, the module $H_{q}(E)_{\mathfrak{m}} / \mathfrak{m} H_{q}(E)_{\mathfrak{m}}$ is also non-zero. Using (11), we conclude that $\mathfrak{m} \in \bigcup_{i=0}^{q} \mathcal{V}_{1}^{i}(E)$, and this completes the proof.

Note that the freeness assumption is again crucial for this theorem to hold. For instance, if $E$ is the chain complex from Example 2.4, then $\bigcup_{i \leq 1} \mathcal{V}_{1}^{i}(E)=\mathbb{k} \backslash\{0\}$, whereas $\bigcup_{i \leq 1} \mathcal{W}_{1}^{i}(E)=\mathbb{k}$.

\section{The resonance varieties of a graded algebra}

We now turn to the resonance varieties associated to a commutative graded algebra, and set up a parameter space where vanishing resonance is a Zariski open condition.

\subsection{Resonance varieties}

Let $A$ be a commutative graded algebra over a field $\mathbb{k}$, for short, a cga. We will assume throughout that $A$ is connected, i.e., $A^{0}=\mathbb{k}$.

Let $a \in A^{1}$, and assume $a^{2}=0$ (this condition is redundant if $\operatorname{char}(\mathbb{k}) \neq$ 2 , by graded-commutativity of the multiplication in $A$ ). The Aomoto complex of $A$ (with respect to $a \in A^{1}$ ) is the cochain complex of $\mathbb{k}$-vector spaces,

$$
(A, \delta(a)): A^{0} \stackrel{\delta^{0}(a)}{\longrightarrow} A^{1} \stackrel{\delta^{1}(a)}{\longrightarrow} A^{2} \stackrel{\delta^{2}(a)}{\longrightarrow \cdots}
$$

with differentials given by $\delta^{i}(a)(b)=a b$, for $b \in A^{i}$. We define the resonance varieties of $A$ as

$$
\mathcal{R}_{d}^{i}(A)=\left\{a \in A^{1} \mid a^{2}=0 \text { and } \operatorname{dim}_{\mathbb{k}} H^{i}(A, \delta(a)) \geq d\right\} .
$$

It follows at once from the definition that $\mathcal{R}_{1}^{0}(A)=\{0\}$ and $\mathcal{R}_{d}^{0}(A)=\emptyset$ for $d>1$, since $A$ is connected.

Lemma 3.1. Suppose $A$ is locally finite, i.e., $\operatorname{dim}_{\mathbb{k}} A^{i}<\infty$, for all $i \geq 1$. Then the sets $\mathcal{R}_{d}^{i}(A)$ are Zariski closed cones inside the affine space $A^{1}$.

Proof. Let $Z=\left\{a \in A^{1} \mid a^{2}=0\right\}$; clearly, $Z$ is a Zariski closed cone in $A^{1}$. By definition, an element $a \in Z$ belongs to the set $\mathcal{R}=\mathcal{R}_{d}^{i}(A)$ if and only if $\operatorname{rank} \delta^{i-1}(a)+\operatorname{rank} \delta^{i}(a) \leq b_{i}-d$, where $b_{i}=\operatorname{dim}_{\mathbb{k}} A^{i}$. An argument as in 
Lemma 2.3 now shows that $\mathcal{R}$ is a Zariski closed subset of $A^{1}$. Clearly, $a \in \mathcal{R}$ if and only if $\lambda a \in \mathcal{R}$, for all $\lambda \in \mathbb{k}^{\times}$; thus, $\mathcal{R}$ is a cone in $A^{1}$.

\subsection{A parameter space for graded algebras}

Let $B=\bigoplus_{i \geq 0} B^{i}$ be a graded $\mathbb{k}$-vector space, with $B^{0}=\mathbb{k}$ and $\operatorname{dim}_{\mathbb{k}} B<\infty$, encoded by the sequence $\left\{b_{i}=\operatorname{dim}_{\mathbb{k}} B^{i}\right\}_{i \geq 1}$. Given these data, we define a parameter space for all commutative graded algebras whose underlying graded vector space is $B$, as follows:

$$
P_{B}=\left\{A \text { a cga } \mid \operatorname{dim}_{\mathbb{k}} A^{i}=\operatorname{dim}_{\mathbb{k}} B^{i} \text { for all } i \geq 0\right\}
$$

It is readily seen that $P_{B}$ is an affine cone in the vector space

$$
V_{B}=\bigoplus_{i, j \geq 1} \operatorname{Hom}_{\mathbb{k}}\left(B^{i} \otimes B^{j}, B^{i+j}\right)
$$

cut out by homogeneous quadrics (corresponding to the associativity conditions for a cga), and linear equations (corresponding to gradedcommutativity conditions for a cga).

Example 3.2. Suppose $B=\mathbb{k} \oplus B^{1} \oplus B^{2}$. In that case, a commutative graded algebra $A \in P_{B}$ corresponds to an anti-symmetric $\mathbb{k}$-linear map $B^{1} \otimes$ $B^{1} \rightarrow B^{2}$. Thus, $P_{B}$ is an affine space; in fact, $P_{B}=\operatorname{Hom}_{\mathbb{k}}\left(B^{1} \wedge B^{1}, B^{2}\right)$ if $\operatorname{char}(\mathbb{k}) \neq 2$.

\subsection{Vanishing resonance}

We are now ready to state and prove the main result of this section.

Theorem 3.3. Let $B$ be a connected, finite-dimensional, graded vector space over an algebraically closed field $\mathbb{k}$. For each $i \geq 1$, let

$$
U_{B}^{i}=\left\{A \in P_{B} \mid \mathcal{R}_{1}^{i}(A) \subseteq\{0\}\right\}
$$

be the set of commutative graded algebras whose underlying graded vector space is $B$ and whose degree $i$ resonance varieties are trivial. Then $U_{B}^{i}$ is a Zariski open subset of $P_{B}$. 
Proof. We need to show that, for each $i \geq 1$, the set

$$
C_{B}^{i}=\left\{A \in P_{B} \mid \text { there is } a \in B^{1} \backslash\{0\} \text { such that } H^{i}(A, \delta(a)) \neq 0\right\}
$$

is Zariski closed. To that end, consider the set

$$
\left\{(A, a) \in P_{B} \times B^{1} \mid a^{2}=0 \text { and } H^{i}(A, \delta(a)) \neq 0\right\} .
$$

A look at the proof of Lemma 3.1 shows that this set is defined by bihomogeneous equations. In particular, the same equations define a Zariski closed subset $T_{B}^{i}$ inside $P_{B} \times \mathbb{P}\left(B^{1}\right)$.

Now consider the first-coordinate projection map $p: P_{B} \times \mathbb{P}\left(B^{1}\right) \rightarrow P_{B}$. Since $\mathbb{P}\left(B^{1}\right)$ is a complete variety, $p$ is a closed map. On the other hand, $p\left(T_{B}^{i}\right)=C_{B}^{i}$, and this completes the proof.

Remark 3.4. The approach from [13], where we consider connected, finitedimensional cgas $A$ over $\mathbb{C}$ with $A^{>2}=0$ and we fix the dimensions of $A^{1}$ and $\operatorname{ker}\left(A^{1} \wedge A^{1} \rightarrow A^{2}\right)$, shows exactly when the vanishing resonance condition in degree 1 is generic. Theorem 1.1 from [13] implies that $U_{B}^{1} \neq \emptyset$ if and only if $b_{2} \geq 2 b_{1}-3$, for an arbitrary connected, finite-dimensional graded $\mathbb{C}$-vector space $B$.

\section{The characteristic and resonance varieties of a CW-complex}

In this section, we present a topological context in which the support varieties, homology jump loci, and resonance varieties arise, and reduce their computation to the case of finite CW-complexes.

\subsection{Homology with twisted coefficients}

Let $X$ be a connected $\mathrm{CW}$-complex. Without loss of generality, we may assume $X$ has a single 0 -cell, call it $x_{0}$. Let $\pi=\pi_{1}\left(X, x_{0}\right)$ be the fundamental group of $X$, based on $x_{0}$.

Let $p: \widetilde{X} \rightarrow X$ be the universal cover of our CW-complex. The cell structure on $X$ lifts to a cell structure on $\widetilde{X}$. Fixing a lift $\tilde{x}_{0} \in p^{-1}\left(x_{0}\right)$ identifies the fundamental group of $X$ with the group of deck transformations of $\widetilde{X}$, which permute the cells. Therefore, we may view the cellular cell complex $C \bullet(\tilde{X}, \mathbb{Z})$, with differential $\tilde{\partial}$, as a chain complex of free left modules over the group-ring $\mathbb{Z} \pi$. 
Given a right $\mathbb{Z} \pi$-module $M$, consider the chain complex $C_{\bullet}(X, M):=$ $M \otimes_{\mathbb{Z} \pi} C_{\bullet}(\tilde{X}, \mathbb{Z})$, with differential id $\operatorname{id}_{M} \otimes \tilde{\partial}$. The homology groups of $X$ with coefficients in $M$ are then defined as $H_{i}(X, M):=H_{i}\left(C_{\bullet}(X, M)\right)$.

Noteworthy is the following situation. Let $X^{\mathrm{ab}} \rightarrow X$ be the universal abelian cover of $X$, with group of deck transformations $\pi_{\mathrm{ab}}=H_{1}(X, \mathbb{Z})$. The cellular chain complex $\left(C_{\bullet}\left(X^{\mathrm{ab}}, \mathbb{Z}\right), \partial\right)$ is a chain complex of free modules over the commutative ring $\mathbb{Z}\left[\pi_{\mathrm{ab}}\right]$. The homology groups $H_{i}\left(X^{\mathrm{ab}}, \mathbb{Z}\right)=$ $H_{i}\left(X, \mathbb{Z}\left[\pi_{\mathrm{ab}}\right]\right)$ are $\mathbb{Z}\left[\pi_{\mathrm{ab}}\right]$-modules, called the Alexander invariants of $X$.

\subsection{Homology jump loci}

Fix a field $\mathbb{k}$, and let $\operatorname{Hom}\left(\pi, \mathbb{k}^{\times}\right)$be the group of characters of $\pi$, with values in the multiplicative group of units of $\mathbb{k}$. Since $\mathbb{k}^{\times}$is an abelian group, every character factors through the abelianization $\pi_{\mathrm{ab}}$, and so we may identify $\operatorname{Hom}\left(\pi, \mathbb{k}^{\times}\right)$with $\operatorname{Hom}\left(\pi_{\mathrm{ab}}, \mathbb{k}^{\times}\right)$. Given a homomorphism $\rho: \pi \rightarrow \mathbb{k}^{\times}$, let $\mathbb{k}_{\rho}$ be the rank 1 local system on $X$ defined by $\rho$, and let $H_{*}\left(X, \mathbb{k}_{\rho}\right)$ be the resulting twisted homology groups.

There are three types of (co)homology jumping loci traditionally associated to a CW-complex $X$ as above. First, the characteristic varieties of $X$ (with coefficients in $\mathbb{k}$ ) are the sets

$$
\mathcal{V}_{d}^{i}(X, \mathbb{k})=\left\{\rho \in \operatorname{Hom}\left(\pi, \mathbb{k}^{\times}\right) \mid \operatorname{dim}_{\mathbb{k}} H_{i}\left(X, \mathbb{k}_{\rho}\right) \geq d\right\} .
$$

Second, the Alexander varieties of $X$ (with coefficients in $\mathbb{k}$ ) are the supports of the exterior powers of the Alexander invariants of $X$,

$$
\mathcal{W}_{d}^{i}(X, \mathbb{k})=\operatorname{supp}\left(\bigwedge^{d} H_{i}\left(X^{\mathrm{ab}}, \mathbb{k}\right)\right) .
$$

And finally, the resonance varieties of $X$ (with coefficients in $\mathbb{k}$ ) are the jumping loci associated to the cohomology algebra $H^{*}(X, \mathbb{k})$,

$$
\mathcal{R}_{d}^{i}(X, \mathbb{k})=\left\{a \in H^{1}(X, \mathbb{k}) \mid a^{2}=0 \text { and } \operatorname{dim}_{\mathbb{k}} H^{i}\left(H^{*}(X, \mathbb{k}), \delta(a)\right) \geq d\right\} .
$$

In absolute generality, there is not much structure on these sets. To remedy this situation, we need to impose some finiteness restrictions on $X$ in order to be able to say more about its jump loci. To start with, let us assume that $X$ has finite 1 -skeleton, and $\mathbb{k}$ is algebraically closed. Then the fundamental group $\pi=\pi_{1}\left(X, x_{0}\right)$ is finitely generated, and the character group $\operatorname{Hom}\left(\pi, \mathbb{k}^{\times}\right)$is an affine algebraic group, with coordinate ring the group algebra of the abelianization, $S=\mathbb{k}\left[\pi_{\mathrm{ab}}\right]$. 
Every character $\rho: \pi_{\mathrm{ab}} \rightarrow \mathbb{k}^{\times}$extends $\mathbb{k}^{-l i n e a r l y}$ to a ring morphism, $\bar{\rho}: \mathbb{k}\left[\pi_{\mathrm{ab}}\right] \rightarrow \mathbb{k}$, and thus gives rise to a maximal ideal $\mathfrak{m}=\operatorname{ker}(\bar{\rho})$ of $S$. Conversely, since $\mathbb{k}$ is algebraically closed, each maximal ideal $\mathfrak{m} \in S$ determines a character $\rho: \pi_{\mathrm{ab}} \rightarrow \mathbb{k}^{\times}$. Thus,

$$
\operatorname{Spec}\left(\mathbb{k}\left[\pi_{\mathrm{ab}}\right]\right)=\operatorname{Hom}\left(\pi_{\mathrm{ab}}, \mathbb{k}^{\times}\right)=\operatorname{Hom}\left(\pi, \mathbb{k}^{\times}\right) .
$$

Let $C_{\bullet}=\left(C_{\bullet}\left(X^{\mathrm{ab}}, \mathbb{k}\right), \partial\right)$ be the equivariant chain complex of the universal abelian cover, with coefficients in $\mathbb{k}$. It is clear from the definitions that $\mathcal{V}_{d}^{i}(X, \mathbb{k})=\mathcal{V}_{d}^{i}\left(C_{\bullet}\right)$ and $\mathcal{W}_{d}^{i}(X, \mathbb{k})=\mathcal{W}_{d}^{i}\left(C_{\bullet}\right)$.

Now suppose that $X$ has finite $k$-skeleton, for some $k \geq 1$. Then, for each $i<k$ and $d>0$, the sets $\mathcal{W}_{d}^{i}(X, \mathbb{k})$ and $\mathcal{V}_{d}^{i}(X, \mathbb{k})$ are Zariski closed subsets of the character group $\operatorname{Hom}\left(\pi, \mathbb{k}^{\times}\right)$, either by definition, for the former, or by Lemma 2.3, for the latter. Likewise, the sets $\mathcal{R}_{d}^{i}(X, \mathbb{k})$ are Zariski closed subsets of the affine space $H^{1}(X, \mathbb{k})$, by Lemma 3.1. In fact, as we shall see next, these statements also hold for $i=k$.

\subsection{Reducing to the finite-dimensional case}

For the purpose of computing resonance varieties or homology with certain twisted coefficients, in the finiteness range of $X$, the following proposition allows us to replace $X$ by a finite $\mathrm{CW}$-complex.

Proposition 4.1. Let $X$ be a connected $C W$-complex with finite $k$-skeleton, for some $k \geq 1$. There exists then a finite $C W$-complex $Y$ of dimension at most $k+1$, with $Y^{(k)}=X^{(k)}$, and a map $f: Y \rightarrow X$ such that the following hold:

(1) The induced homomorphism, $f_{*}: H_{1}(Y, \mathbb{Z}) \rightarrow H_{1}(X, \mathbb{Z}), \quad$ is an isomorphism.

(2) For every commutative ring $R$, and for every homomorphism $\rho: H_{1}(X, \mathbb{Z}) \rightarrow R^{\times}$, the induced homomorphism, $f_{*}: H_{i}\left(Y, R_{\rho \circ f_{*}}\right) \rightarrow$ $H_{i}\left(X, R_{\rho}\right)$, is an $R$-module isomorphism, for all $i \leq k$.

(3) The isomorphism $f^{*}: \operatorname{Hom}\left(H_{1}(X, \mathbb{Z}), \mathbb{k}^{\times}\right) \rightarrow \operatorname{Hom}\left(H_{1}(Y, \mathbb{Z}), \mathbb{k}^{\times}\right)$ restricts to isomorphisms from $\mathcal{W}_{d}^{i}(X, \mathbb{k})$ to $\mathcal{W}_{d}^{i}(Y, \mathbb{k})$, for all $i \leq k$.

(4) The isomorphism $f^{*}: \operatorname{Hom}\left(H_{1}(X, \mathbb{Z}), \mathbb{k}^{\times}\right) \rightarrow \operatorname{Hom}\left(H_{1}(Y, \mathbb{Z}), \mathbb{k}^{\times}\right)$ restricts to isomorphisms from $\mathcal{V}_{d}^{i}(X, \mathbb{k})$ to $\mathcal{V}_{d}^{i}(Y, \mathbb{k})$, for all $i \leq k$.

(5) The isomorphism $f^{*}: H^{1}(X, \mathbb{k}) \rightarrow H^{1}(Y, \mathbb{k})$ restricts to isomorphisms from $\mathcal{R}_{d}^{i}(X, \mathbb{k})$ to $\mathcal{R}_{d}^{i}(Y, \mathbb{k})$, for all $i \leq k$. 
Proof. The cellular chain complex $\left(C_{i}\left(X^{\mathrm{ab}}\right), \partial_{i}^{\mathrm{ab}}\right)_{i \geq 0}$ is a chain complex of free modules over the (commutative) Noetherian ring $S=\mathbb{Z}\left[\pi_{\mathrm{ab}}\right]$. Since $C_{k}\left(X^{\mathrm{ab}}\right)$ is finitely generated as an $S$-module, the $S$-submodule $B_{k}\left(X^{\mathrm{ab}}\right)=$ $\operatorname{im}\left(\partial_{k+1}^{\mathrm{ab}}\right)$ is also finitely generated, let us say, by the images of the $(k+1)$ cells $e_{1}, \ldots, e_{r}$. Set

$$
Z:=X^{(k)} \cup e_{1} \cup \cdots \cup e_{r}
$$

Clearly, $Z$ is a finite subcomplex of $X^{(k+1)}$; let $g: Z \rightarrow X$ be the inclusion map. Consider the cup-product map, $\cup_{X}^{k+1}: H^{1}(X, \mathbb{k}) \otimes H^{k}(X, \mathbb{k}) \rightarrow$ $H^{k+1}(X, \mathbb{k})$. Passing to $\mathbb{k}$-duals, we obtain the comultiplication map, $\nabla_{k+1}^{X}$ : $H_{k+1}(X, \mathbb{k}) \rightarrow H_{1}(X, \mathbb{k}) \otimes H_{k}(X, \mathbb{k})$.

The dual of $\operatorname{im}\left(\cup_{X}\right)$ may be identified with $\operatorname{im}\left(\nabla_{X}\right)$. Proceeding in the same fashion with the space $Z$, and comparing the resulting maps via the induced homomorphism $g_{*}: H_{*}(Z, \mathbb{k}) \rightarrow H_{*}(X, \mathbb{k})$, we obtain the following commuting diagram:

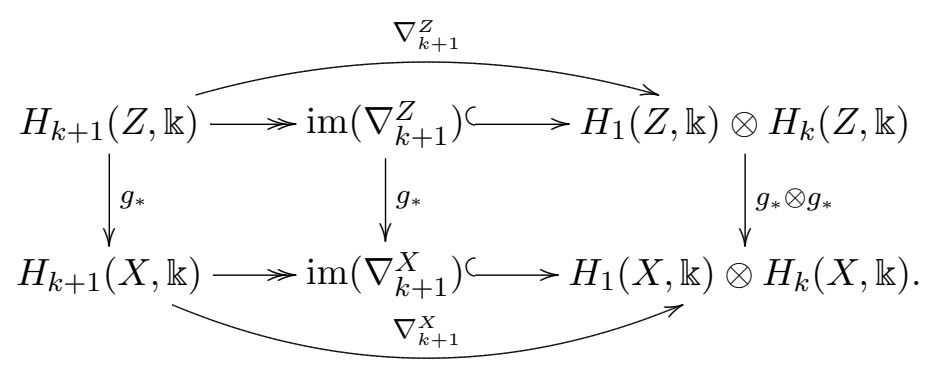

In general, the vertical arrow in the center of the above diagram is not surjective. So pick a (finite) $\mathbb{k}$-basis, $x_{1}, \ldots, x_{s}$ for $\operatorname{im}\left(\nabla_{k+1}^{X}\right)$, lift those homology classes back to $y_{1}=\left[z_{1}\right], \ldots, y_{s}=\left[z_{s}\right] \in H_{k+1}(X, \mathbb{k})$, and represent the cycles $z_{1}, \ldots, z_{s}$ as $\mathbb{k}$-linear combinations of finitely many $(k+1)$-cells of $X$, say, $e_{r+1}, \ldots, e_{t}$. Then, the CW-complex

$$
Y:=Z \cup e_{r+1} \cup \cdots \cup e_{t}
$$

is again a finite subcomplex of $X^{(k+1)}$; let $f: Y \rightarrow X$ be the inclusion map. Redrawing diagram (23) with $Z$ replaced by $Y, g_{*}$ by $f_{*}$, etc, we see that the middle arrow is now surjective. Hence, the dual map, $f^{*}: \operatorname{im}\left(\cup_{X}^{k+1}\right) \rightarrow$ $\operatorname{im}\left(\cup_{Y}^{k+1}\right)$, is injective.

Clearly, $f_{*}: H_{1}(Y, \mathbb{Z}) \rightarrow H_{1}(X, \mathbb{Z})$ is an isomorphism, thus proving part (1), and showing that the maps $f^{*}$ in parts (3)-(5) are also isomorphisms.

By construction, the map $f_{*}: H_{i}(Y, R) \rightarrow H_{i}(X, R)$ from part (2) is an $R$-isomorphism, for all $i \leq k$, since $\rho$ corresponds to a change-of-rings map, 
$S \rightarrow R$. Furthermore, parts (3) and (4) are now a direct consequence of part (2).

Also by construction, the map $f^{*}: H^{1}(X, \mathbb{k}) \rightarrow H^{1}(Y, \mathbb{k})$ restricts to isomorphisms $\mathcal{R}_{d}^{i}(X, \mathbb{k}) \stackrel{\simeq}{\longrightarrow} \mathcal{R}_{d}^{i}(Y, \mathbb{k})$, for all $i \leq k$ and all $d>0$. This finishes the proof of part (5).

The following corollary is now immediate.

Corollary 4.2. Let $X$ be a connected $C W$-complex with finite $k$-skeleton, for some $k \geq 1$. Then, for each $i \leq k$ and $d>0$, the sets $\mathcal{W}_{d}^{i}(X, \mathbb{k})$ and $\mathcal{V}_{d}^{i}(X, \mathbb{k})$ are subvarieties of the character group $\operatorname{Hom}\left(\pi, \mathbb{k}^{\times}\right)$, while the sets $\mathcal{R}_{d}^{i}(X, \mathbb{k})$ are subvarieties of the affine space $H^{1}(X, \mathbb{k})$.

Using Theorem 2.5 and Proposition 4.1, we obtain the following result, which recovers Corollary 3.7 from [11].

Corollary 4.3. Let $X$ be a connected $C W$-complex with finite $k$-skeleton, for some $k \geq 1$. Then, for all integers $q \leq k$,

$$
\bigcup_{i \leq q} \mathcal{V}_{1}^{i}(X, \mathbb{k})=\bigcup_{i \leq q} \mathcal{W}_{1}^{i}(X, \mathbb{k})
$$

\section{The equivariant spectral sequence}

We now relate the homology jump loci associated to the first page of the equivariant spectral sequence of a space to the resonance varieties of its cohomology algebra.

\subsection{The spectrum of the associated graded ring of a group ring}

Let $G$ be a finitely generated abelian group, and let $\mathbb{k} G$ be the group ring over an algebraically closed field $\mathbb{k}$. The powers of the augmentation ideal, $J=\operatorname{ker}(\mathbb{k} G \stackrel{\epsilon}{\rightarrow} \mathbb{k})$, define a descending filtration on $\mathbb{k} G$. The associated graded ring, $S^{\bullet}=\operatorname{gr}_{J}^{\cdot}(\mathbb{k} G)$, is an affine $\mathbb{k}$-algebra, whose maximal spectrum (endowed with the Zariski topology) we denote by $\operatorname{Spec}(S)$.

Let $\bar{G}=G / \operatorname{tors}(G)$ be the maximal torsion-free quotient of $G$, and let $\bar{J}$ be the augmentation ideal of $\mathbb{k} \bar{G}$. The associated graded $\operatorname{ring}, \bar{S}=\operatorname{gr}_{\bar{J}}(\mathbb{k} \bar{G})$, may be identified with the polynomial ring $\mathbb{k}\left[x_{1}, \ldots, x_{r}\right]$, where $r=\operatorname{rank}(\bar{G})$. Consequently, $\operatorname{Spec}(\bar{S})$ may be identified with the affine space $H^{1}$ $(\bar{G}, \mathbb{k})=\mathbb{k}^{r}$. 
The natural projection $\phi: G \rightarrow \bar{G}$ extends to a ring epimorphism $\phi: S \rightarrow$ $\bar{S}$. In general, this morphism is not injective. For instance, if char $\mathbb{k}=p$ and $G=\mathbb{Z} / p^{s} \mathbb{Z}$, then $\mathbb{k} G=\mathbb{k}[t] /\left(t^{p^{s}}-1\right)=\mathbb{k}[t] /(t-1)^{p^{s}}$, and thus $S=\mathbb{k}[x] /$ $\left(x^{p^{s}}\right)$, whereas $\bar{S}=\mathbb{k}$. Passing to maximal spectra, though, fixes this fat point issue.

Let $\operatorname{Nil}(S)$ be the nilradical of the ring $S$, i.e., the intersection of all its prime ideals.

Lemma 5.1. The kernel of the epimorphism $\phi: S \rightarrow \bar{S}$ is equal to $\operatorname{Nil}(S)$. Consequently, $\phi$ induces a homeomorphism $\operatorname{Spec}(\bar{S}) \rightarrow \operatorname{Spec}(S)$.

Proof. First suppose that $G=G_{1} \times G_{2}$. Recall that the group ring of a direct product of groups is canonically isomorphic to the tensor product of the group rings of the factors. Thus, we have an isomorphism of $\mathbb{k}$-algebras, $\alpha: \mathbb{k} G_{1} \otimes_{\mathbb{k}} \mathbb{k} G_{2} \stackrel{\simeq}{\longrightarrow} \mathbb{k} G$. Now let $J \subset \mathbb{k} G$ and $J_{i} \subset \mathbb{k} G_{i}$ be the respective augmentation ideals. A standard inductive argument shows that

$$
\alpha\left(\sum_{s+t=n} J_{1}^{s} \otimes J_{2}^{t}\right)=J^{n}
$$

for all $n \geq 0$. Hence, the map $\alpha$ induces an isomorphism of (connected) graded $\mathbb{k}$-algebras, $\operatorname{gr}(\alpha): S_{\dot{1}} \otimes_{\mathbb{k}} S_{\dot{2}} \stackrel{\simeq}{\longrightarrow} S \cdot$. Applying the same argument to the decomposition $\bar{G}=\bar{G}_{1} \times \bar{G}_{2}$, we obtain an isomorphism $\operatorname{gr}(\bar{\alpha}): \bar{S}_{1} \otimes_{\mathbb{k}}$ $\bar{S}_{2} \stackrel{\simeq}{\longrightarrow} \bar{S} \cdot$ which fits into the commuting diagram

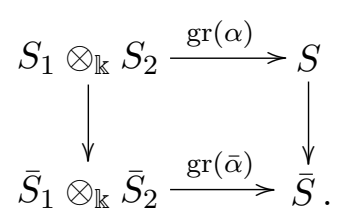

Taking $G_{1}=\bar{G}$ and $G_{2}=\operatorname{Tors}(G)$, we infer from (26) that $\phi$ may be identified with id $\otimes \epsilon: \bar{S} \otimes S_{2} \rightarrow \bar{S} \otimes \mathbb{k}$. In particular, $\operatorname{ker}(\phi)$ is identified with $\bar{S} \otimes S_{2}^{+}$.

Plainly, $\operatorname{Nil}(\bar{S})=0$, and so $\operatorname{Nil}\left(\bar{S} \otimes S_{2}\right) \subseteq \operatorname{ker}(\phi)$. We have to prove the reverse inclusion. Note that $S_{2}$ is a tensor product of graded algebras of the form $R \cdot=\operatorname{gr}_{J}^{\cdot} \mathbb{k}\left(\mathbb{Z} / p^{s} \mathbb{Z}\right)$, for some prime $p$ and integer $s \geq 1$. Clearly, it is enough to show that $R^{+} \subseteq \operatorname{Nil}(R)$.

If $p \neq \operatorname{char}(\mathbb{k})$, then $R^{1}=\left(\mathbb{Z} / p^{s} \mathbb{Z}\right) \otimes \mathbb{k}$ vanishes, and thus $R^{+}=0$. If $p=\operatorname{char}(\mathbb{k})$, then $R=\mathbb{k}[x] /\left(x^{p^{s}}\right)$, and thus $R^{+} \subseteq \operatorname{Nil}(R)$. In either case, the desired conclusion holds, and we are done. 


\subsection{The first page of the equivariant spectral sequence}

Let $X$ be a connected, finite-type CW-complex and fix a coefficient field $\mathbb{k}$. As before, let $\cup_{X}$ be the cup-product map in $H^{*}(X, \mathbb{k})$, and let $\nabla^{X}$ be the co-multiplication map in $H_{*}(X, \mathbb{k})$.

Next, let $\nu: \pi \rightarrow G$ be an epimorphism from the fundamental group $\pi=$ $\pi_{1}\left(X, x_{0}\right)$ to an abelian group $G$ (necessarily, $G$ must be finitely generated). Let $\mathbb{k} G$ be the group ring of $G$, and let $\widehat{\mathbb{k} G}$ be the completion of this ring with respect to the filtration by powers of the augmentation ideal. Composing the completion map $\mathbb{k} G \rightarrow \widehat{\mathbb{k} G}$ with the extension of $\nu$ to group rings yields a ring morphism, $\hat{\nu}: \mathbb{k} \pi \rightarrow \widehat{\mathbb{k} G}$. Clearly, this morphism makes the completion $\widehat{\mathbb{k} G}$ into a module over $\mathbb{k} \pi$.

Following the setup from [10], let

$$
E=\left(E^{1}(X, \widehat{\mathbb{K} G}), d^{1}\right)
$$

be the first page of the equivariant spectral sequence of $X$ with coefficients in $\widehat{\mathbb{k} G}$. This is a chain complex of free, finitely generated modules over the affine algebra $S=\operatorname{gr}_{J}(\mathbb{k} G)$. The $i$ th term of this chain complex is

$$
E_{i}=S \otimes_{\mathbb{k}} H_{i}(X, \mathbb{k})
$$

while the $i$ th differential, $d_{i}^{1}: E_{i} \rightarrow E_{i-1}$, when restricted to the generating set $\{1\} \otimes H_{i}(X, \mathbb{k})$, is the composite

$d_{i}^{1}: H_{i}(X, \mathbb{k}) \stackrel{\nabla_{i}^{X}}{\longrightarrow} H_{1}(X, \mathbb{k}) \otimes_{\mathbb{k}} H_{i-1}(X, \mathbb{k}) \stackrel{\nu_{*} \otimes \mathrm{id}}{\longrightarrow} H_{1}(G, \mathbb{k}) \otimes_{\mathbb{k}} H_{i-1}(X, \mathbb{k})$.

By definition, the transpose of $\nabla_{i}^{X}$ is the cup-product map $\cup_{X}^{i}$. Thus, the transpose of $d_{i}^{1}$ is the composite

$$
\left(d_{i}^{1}\right)^{\top}: H^{1}(G, \mathbb{k}) \otimes_{\mathbb{k}} H^{i-1}(X, \mathbb{k}) \stackrel{\nu^{*} \otimes \mathrm{id}}{\longrightarrow} H^{1}(X, \mathbb{k}) \otimes_{\mathbb{k}} H^{i-1}(X, \mathbb{k}) \stackrel{\cup_{X}^{i}}{\longrightarrow} H^{i}(X, \mathbb{k}) .
$$

\subsection{Homology jump loci and resonance varieties}

Now assume $\mathbb{k}$ is algebraically closed. By Lemma 5.1, we may identify the maximal spectrum $\operatorname{Spec}(S)$ with $H^{1}(\bar{G}, \mathbb{k})$, where recall $\bar{G}$ is the maximal torsion-free quotient of $G$.

Using this identification, we may view the homology jump loci $\mathcal{V}_{d}^{i}(E)$ of the chain complex $E$ from $(27)$ as subsets of the $\mathbb{k}$-vector space $H^{1}(\bar{G}, \mathbb{k})$. 
The next result compares these loci with the resonance varieties of $X$, viewed as subsets of $H^{1}(X, \mathbb{k})=H^{1}(\pi, \mathbb{k})$.

Theorem 5.2. Let $\nu: \pi \rightarrow G$ be an epimorphism onto an abelian group, and set $\bar{\nu}=\phi \circ \nu: \pi \rightarrow \bar{G}$. Let $w \in H^{1}(\bar{G}, \mathbb{k})$. Then

$$
w \in \mathcal{V}_{d}^{i}(E) \Longleftrightarrow \bar{\nu}^{*}(w) \in \mathcal{R}_{d}^{i}(X, \mathbb{k}) .
$$

Proof. By definition, an element $a \in H^{1}(X, \mathbb{k})$ belongs to $\mathcal{R}_{d}^{i}(X, \mathbb{k})$ if and only if $a^{2}=0$ and $\operatorname{dim}_{\mathbb{k}} H^{i}\left(H^{*}(X, \mathbb{k}), \delta(a)\right) \geq d$, where recall $\delta^{i}(a)$ : $H^{i}(X, \mathbb{k}) \rightarrow H^{i+1}(X, \mathbb{k})$ is left-multiplication by $a$.

Now let $w \in H^{1}(\bar{G}, \mathbb{k})$. Clearly, $w^{2}=0$, and thus $\bar{\nu}^{*}(w)^{2}=0$. Let $\rho: S \rightarrow$ $\mathbb{k}$ be the $\mathbb{k}$-algebra morphism defined by $\phi^{*}(w) \in \operatorname{Spec}(S)$. Denote by $d^{1}(w)$ the specialization of $d^{1}$ at $\rho$. Using sequence (30), we find that

$$
\left(d_{i}^{1}(w)\right)^{\top}=\delta^{i-1}\left(\bar{\nu}^{*}(w)\right) .
$$

Therefore,

$$
\begin{aligned}
w \in \mathcal{V}_{d}^{i}(E) & \Longleftrightarrow \operatorname{dim}_{\mathbb{k}} H_{i}\left(H_{*}(X, \mathbb{k}), d^{1}(w)\right) \geq d \\
& \Longleftrightarrow \operatorname{dim}_{\mathbb{k}} H^{i}\left(H^{*}(X, \mathbb{k}),\left(d^{1}(w)\right)^{\top}\right) \geq d \\
& \Longleftrightarrow \bar{\nu}^{*}(w) \in \mathcal{R}_{d}^{i}(X, \mathbb{k})
\end{aligned}
$$

and this completes the proof.

Remark 5.3. Noteworthy is the situation when $\pi_{\mathrm{ab}}$ is torsion free and $\nu$ is the abelianization map, ab: $\pi \rightarrow \pi_{\mathrm{ab}}$, in which case $H^{1}\left(\bar{\pi}_{\mathrm{ab}}, \mathbb{k}\right)=H^{1}(X, \mathbb{k})$. Let $E$ be the first page of the corresponding spectral sequence. Applying Theorem 5.2, we may then identify $\mathcal{V}_{d}^{i}(E)$ with $\mathcal{R}_{d}^{i}(X, \mathbb{k})$.

Remark 5.4. It would be interesting to find a similar interpretation for the homology jumping loci of the other pages in the equivariant spectral sequence. Such an interpretation would likely involve the higher-order Massey products in $H^{*}(X, \mathbb{k})$. This is suggested by the case $G=\mathbb{Z}$, when, as explained in [10, Section 1.4], our equivariant spectral sequence is related to the Farber-Novikov spectral sequence, whose differentials are given by certain Massey products, see for instance [7, page 192].

\section{Jump loci and finiteness properties}

In this section, we relate the vanishing of the resonance varieties of a space to the finiteness properties of its completed Alexander-type invariants. First, we 
need to recall a well-known fact from commutative algebra. For the reader's convenience, we give a sketch of a proof (see also [15, Proposition 9.3]).

Lemma 6.1. Let $\mathbb{k}$ be an algebraically closed field, and let $M$ be a finitely generated module over an affine $\mathbb{k}$-algebra $S$. Then

$$
\operatorname{dim}_{\mathbb{k}} M<\infty \Longleftrightarrow \operatorname{supp} M \text { is finite. }
$$

Proof. The module $M$ is finite dimensional over $\mathbb{k}$ if and only if $M$ has finite length. This condition is equivalent to $M$ having 0 Krull dimension, i.e., $\operatorname{dim}(S / \operatorname{ann}(M))=0$. In turn, this means that the support of $M$ is 0 dimensional, i.e., $\operatorname{supp} M$ is finite.

Now let $X$ be a connected CW-complex with finite $k$-skeleton, for some $k \geq 1$. Given an epimorphism $\nu: \pi \rightarrow G$ from $\pi=\pi_{1}\left(X, x_{0}\right)$ to a (finitely generated) abelian group $G$, let $\phi: G \rightarrow \bar{G}$ be the projection onto the maximal free-abelian quotient of $G$, and set $\bar{\nu}=\phi \circ \nu$.

Let $\mathbb{k} G$ be the group-ring of $G$, with coefficients in an algebraically closed field $\mathbb{k}$, let $J$ be its augmentation ideal, and let $\widehat{\mathbb{k} G}$ be the $J$-adic completion of $\mathbb{k} G$. As shown in Lemma 5.1, the associated graded $\operatorname{ring} S=\operatorname{gr}_{J}(\mathbb{k} G)$ is an affine $\mathbb{k}$-algebra, whose maximal spectrum $\operatorname{Spec}(S)$ may be identified with $H^{1}(\bar{G}, \mathbb{k})$.

Theorem 6.2. Let $X^{\nu} \rightarrow X$ be the Galois cover associated to an epimorphism $\nu: \pi \rightarrow G$, and let $\widehat{\left.H_{*} \widehat{\left(X^{\nu},\right.}, \mathbb{k}\right)}$ be the J-adic completion of the $\mathbb{k} G$ module $H_{*}\left(X^{\nu}, \mathbb{k}\right)=H_{*}\left(X,(\mathbb{k} G)_{\nu}\right)$. Then

$$
\left.\bar{\nu}^{*}\left(H^{1}(\bar{G}, \mathbb{k})\right) \cap\left(\bigcup_{0 \leq i \leq k} \mathcal{R}_{1}^{i}(X, \mathbb{k})\right)=\{0\} \Longrightarrow \operatorname{dim}_{\mathbb{k}} \bigoplus_{0 \leq i \leq k} \widehat{H_{i}} \widehat{\left(X^{\nu}, \mathbb{k}\right.}\right)<\infty
$$

Proof. By Proposition 4.1, we may assume $X$ is a finite complex. We use the equivariant spectral sequence $E^{\bullet}(X, \widehat{\mathbb{k} G})$ associated to the Galois cover $X^{\nu} \rightarrow X$ corresponding to the epimorphism $\nu: \pi_{1}(X) \rightarrow G$, with coefficients given by the ring morphism $\hat{\nu}: \mathbb{k} \pi_{1}(X) \rightarrow \widehat{\mathbb{k} G}$. 
The first page of the spectral sequence is $\left(E^{1}, d^{1}\right)$, a chain complex of free, finitely generated $S$-modules, with $E^{1}=S \otimes_{\mathbb{k}} H_{*}(X, \mathbb{k})$. We then have

$$
\begin{aligned}
\bar{\nu}^{*}\left(H^{1}(\bar{G}, \mathbb{k})\right) \cap\left(\bigcup_{i \leq k} \mathcal{R}_{1}^{i}(X, \mathbb{k})\right) & =\bar{\nu}^{*}\left(\bigcup_{i \leq k} \mathcal{V}_{1}^{i}\left(E^{1}\right)\right) \quad \text { by Theorem } 5.2 \\
& =\bar{\nu}^{*}\left(\bigcup_{i \leq k} \mathcal{W}_{1}^{i}\left(E^{1}\right)\right) \quad \text { by Theorem } 2.5 \\
& =\bar{\nu}^{*}\left(\operatorname{supp}\left(\bigoplus_{i \leq k} H_{i}\left(E^{1}\right)\right)\right) \\
& =\bar{\nu}^{*}\left(\operatorname{supp}\left(\bigoplus_{i \leq k} E_{i}^{2}\right)\right) .
\end{aligned}
$$

Using our assumption and the injectivity of $\bar{\nu}^{*}$, we conclude that the support of $\bigoplus_{i<k} E_{i}^{2}$ is the set $\{0\}$. Hence, by Lemma 6.1 , the $\mathbb{k}$-vector space $\bigoplus_{i \leq k} E_{i}^{2}$ is finite dimensional. It follows that $\bigoplus_{i \leq k} E_{i}^{\infty}$ is also finite dimensional.

On the other hand, as shown in [10, Section 8], the equivariant spectral sequence converges to $H_{*} \widehat{\left(X^{\nu}, \mathbb{k}\right)}$, and the spectral sequence filtration on the limit is separated. The desired conclusion readily follows.

Corollary 6.3. If $\mathcal{R}_{1}^{i}(X, \mathbb{k}) \subseteq\{0\}$ for $1 \leq i \leq k$, then $\left.\operatorname{dim}_{\mathbb{k}} H_{i} \widehat{\left(X^{\mathrm{ab}}\right.}, \mathbb{k}\right)<$ $\infty$ for $i \leq k$.

In the particular case when $k=1$ and $\mathbb{k}=\mathbb{C}$, this corollary was proved in [2, Theorem C] by different methods, specific to homological degree 1. It is also shown in [2] that the converse holds in this case, under an additional formality assumption. More precisely, if $X$ is 1 -formal in the sense of Sullivan [16], and the completion of $H_{1}\left(X^{\mathrm{ab}}, \mathbb{C}\right)$ is finite dimensional, then $\mathcal{R}_{1}^{1}(X, \mathbb{C}) \subseteq\{0\}$.

In general, though, the converse to Corollary 6.3 does not hold. For instance, if $X$ is the Heisenberg three-dimensional nilmanifold, then the cupproduct vanishes on $H^{1}(X, \mathbb{C})=\mathbb{C}^{2}$, and so $\mathcal{R}_{1}^{1}(X, \mathbb{C})=\mathbb{C}^{2}$. On the other hand, $X^{\mathrm{ab}} \simeq S^{1}$, and thus $H_{1}\left(X^{\mathrm{ab}}, \mathbb{C}\right)=\mathbb{C}$.

It should also be pointed out that it is really necessary to take the completion of the Alexander invariant in Corollary 6.3, even when $X$ is formal. For instance, if $X$ is the presentation 2-complex for the group 
$\pi=\left\langle a, b \mid a^{2} b=b a^{2}\right\rangle$ from $\left[11\right.$, Example 6.4], we have that $\mathcal{R}_{1}^{1}(X, \mathbb{C})=\{0\}$, yet $\operatorname{dim}_{\mathbb{C}} H_{1}\left(X^{\mathrm{ab}}, \mathbb{C}\right)=\infty$.

For more details on the relationship between the first resonance variety and the first Alexander invariant of a space, we refer to [12]. Further generalizations and applications will be pursued elsewhere.

\section{Acknowledgment}

Papadima was partially supported by the Romanian Ministry of National Education, CNCS-UEFISCDI, grant PNII-ID-PCE-2012-4-0156, and Suciu by NSF grant DMS-1010298 and NSA grant H98230-13-1-0225. This work was started in May-June 2010, when both authors visited the Centro di Ricerca Matematica Ennio De Giorgi in Pisa, and was continued in Spring 2011, when the first author visited Northeastern University. The work was completed while the authors visited the University of Sydney, in December 2012. We are grateful for the support and hospitality provided, and we also thank Krzysztof Kurdyka and Laurenţiu Păunescu for helpful discussions. We are also grateful to the referees, for their pertinent remarks and suggestions.

\section{References}

[1] A. Dimca, R. Hain and S. Papadima, The abelianization of the Johnson kernel, J. Eur. Math. Soc. 16(4) (2014), 805-822.

[2] A. Dimca and S. Papadima, Arithmetic group symmetry and finiteness properties of Torelli groups, Ann of Math. 177(2) (2013), 395-423.

[3] A. Dimca and S. Papadima, Nonabelian cohomology jump loci from an analytic viewpoint, Commun. Contemp. Math. 16(4) (2014), 1350025 (47 pages).

[4] A. Dimca, S. Papadima and A. Suciu, Topology and geometry of cohomology jump loci, Duke Math. J. 148(3) (2009), 405-457.

[5] W.G. Dwyer and D. Fried, Homology of free abelian covers. I, Bull. London Math. Soc. 19(4) (1987), 350-352.

[6] D. Eisenbud, Commutative algebra with a view towards algebraic geometry, Grad. Texts in Math., 150, Springer-Verlag, New York, 1995.

[7] M. Farber, Topology of closed one-forms, Math. Surveys Monogr., 108 Amer. Math. Soc., Providence, RI, 2004. 
[8] M. Green and R. Lazarsfeld, Deformation theory, generic vanishing theorems and some conjectures of Enriques, Catanese and Beauville, Invent. Math. 90(2) (1987), 389-407.

[9] R. Hain, Genus 3 mapping class groups are not Kähler, preprint, arXiv: 1305.2052

[10] S. Papadima and A. Suciu, The spectral sequence of an equivariant chain complex and homology with local coefficients, Trans. Amer. Math. Soc. 362(5) (2010), 2685-2721.

[11] S. Papadima and A. Suciu, Bieri-Neumann-Strebel-Renz invariants and homology jumping loci, Proc. London Math. Soc. 100(3) (2010), 795-834.

[12] S. Papadima and A. Suciu, Homological finiteness in the Johnson filtration of the automorphism group of a free group, J. Topol. 5(4) (2012), 909-944.

[13] S. Papadima and A. Suciu, Vanishing resonance and representations of Lie algebras, J. Reine Angewa. Math. (Crelle's J.), to appear, doi:10.1515/crelle-2013-0073, arXiv: 1207.2038.

[14] A. Suciu, Characteristic varieties and Betti numbers of free abelian covers, Intern. Math. Res. Notices 2014(4) (2014), 1063-1124.

[15] A. Suciu, Y. Yang, G. Zhao, Homological finiteness of abelian covers, Ann. Sc. Norm. Super. Pisa, to appear, at doi:10.2422/20362145.201205_008, available at arXiv:1204.4873.

[16] D. Sullivan, Infinitesimal computations in topology, Inst. Hautes Études Sci. Publ. Math. 47 (1977), 269-331.

Simion Stollow Institute of Mathematics

P.O. Box 1-764

RO-014700 Bucharest

ROMANIA

E-mail address: Stefan.Papadima@imar.ro

Department of Mathematics

NORTHEASTERN UNIVERSiTy

Boston, MA 02115

USA

E-mail address: a.suciu@neu.edu

Received February 22, 2013 
